Ciencia y Educación, Vol. 5, No. 2, mayo-agosto, 2021

ISSN (impreso): 2613-8794 • ISSN (en línea): 2613-8808

DOI: https://doi.org/10.22206/cyed.2021.v5i2.pp63-78

\title{
La neuroeducación en los programas de formación y profesionalización docente en México
}

\author{
Neuroeducation in Mexican' teacher education and \\ professionalization programs
}

Alejandro Díaz-Cabriales ${ }^{a}$ ORCID: 0000-0001-8116-1989

Recibido: $\mathrm{xxx} \bullet$ Aprobado: $\mathrm{xx}$

Cómo citar: Díaz-Cabriales, A. (2021). La neuroeducación en los programas de formación y profesionalización docente en México. Ciencia y Educación, 5(2), 63-78. https://doi.org/10.22206/cyed.2021.v5i2.pp63-78

\section{Resumen}

La necesidad de contar con una base diagnóstica de la presencia de la neuroeducación en los programas de formación y profesionalización docente en México requiere de estudios que aporten conocimiento científico y que contribuyan con elementos para fortalecer la investigación en la neuroeducación. Esta investigación se realizó bajo la metodología cuantitativa con un enfoque no experimental de alcance descriptivo y con diseño transeccional. Se revisaron un total de 2,891 mallas curriculares de programas relacionados con la formación, actualización y profesionalización docente pertenecientes a los niveles de licenciatura y posgrado. Se encontró que el $12 \%$ de los programas incluye materias de neuroeducación en su currículo, siendo 306 de nivel licenciatura, 1 de especialidad, 39 de maestría y ninguno de doctorado. Se observa, además, que las materias predominantes en estos planes de estudio están relacionadas con la neurociencia y con la neuropsicología, demostrando la necesidad de que los programas de formación y actualización docente en México incorporen un mayor número de materias neuroeducativas relacionadas con la neuropedagogía y la neurodidáctica.

Palabras clave: docente, plan de estudios, neuroeducación, normalismo, formación inicial.

\begin{abstract}
Knowing how neuroeducation has been inserted in the teacher's education curricula in Mexico, is a starting point to generate new research on this field. This research is a quantitative work, made under no experimental method, descriptive under a cross-sectional design, the educational institutions that were studied are included in the database of Asociación Nacional de Universidades e Instituciones de Educación Superior (ANUIES). Analyzing 2,891 programs was found that $12 \%$ of educational programs (306 undergraduate, 1 specialization, 39 masters and nondoctoral program) related to teacher education in México have at least one neuroeducation subject in their curricula.
\end{abstract}

Keywords: Teachers, study plan, neuroeducation, normal education, initial training.

a Centro de Investigación e Innovación para el Desarrollo Educativo (CIIDE), México. Correo-e: alejandro.diazc@durango.gob.mx 


\section{Introducción}

La formación inicial de maestros y maestras en México está sufriendo cambios acelerados derivados de las influencias pedagógicas que se generan en el mundo, respondiendo además a las necesidades educativas de los niños y niñas del país que serán quienes tengan el impacto directo de las acciones que se tomen dentro de las aulas normalistas respecto a la preparación de los futuros docentes. Es por lo anterior y ante el avance de la ciencia que surge la necesidad de unirse a la tendencia que ha iniciado desde hace ya casi cuatro décadas de unir la educación con la ciencia, en específico con aquellas disciplinas científicas que se encargan del estudio del cerebro y su funcionamiento (Sánchez, Castro, Casas y Vallejos, 2016).

Este trabajo tiene como objetivo conocer la presencia de la neuroeducación en los planes y programas de estudio para la formación y profesionalización de docentes en México, ya que:

Se requiere un perfil docente que estudie y tenga un conocimiento elemental de la estructura del cerebro, que pueda hacer puente y vincular los aportes neurocientíficos con la práctica pedagógica, el docente debe ser un profesional cualificado capaz de entablar diálogo interdisciplinar entre la neurociencia y la práctica pedagógica (Pherez, Vargas y Jeréz, 2018, p. 158).

Es entonces necesario que los maestros puedan desarrollar habilidades de planeación bajo los principios de la neuroeducación, usando a su favor los conocimientos sobre los principios de aprendizaje compatible con el cerebro, el diseńo instruccional, el diseño universal de aprendizaje, el aprendizaje significativo y la modificabilidad cognitiva estructural, entre otros campos de estudio relacionados con la neurociencia cognitiva aplicada.

Los aportes que la neurociencia ha puesto a disposición de la educación y de los estudios tanto ontogenéticos como filogenéticos permiten que el fenómeno del aprendizaje y del desarrollo cognitivo del ser humano sea exponencial. El estudio de los aportes neurodidácticos posibilita el conocimiento de cómo aprende y se desarrolla el órgano más importante del ser humano: el cerebro. De la misma manera ofrece la posibilidad de estudiar de una manera más objetiva aquellas barreras que afectan el aprendizaje de los alumnos con problemas, "esto es clave no sólo para intervenir en su recuperación de forma eficaz, sino también para ayudar a la prevención de estos, e integrar los nuevos conocimientos en la educación en general" (D’Addario, 2019, p. 9).

Durante el desarrollo de las disciplinas científicas que han surgido de la unión entre la neurociencia, la psicología y la pedagogía han aparecido diversos campos de estudio como la neurodidáctica (Forés $\&$ Ligioiz, 2009; D’Addario, 2019), la neuropsicología (Martín-Lobo, 2016; MECD, 2016), la neuropedagogía (Calzadilla, 2017; Ferreira, 2012), la neuropsicopedagogía (Varela et al., 2011) y la neuroeducación (D’Addario, 2019), que según Nouri \& Mehrmohammadi (2013) ponen de manifiesto la urgencia de alejarse de los neuromitos con el fin de lograr un aprendizaje que modificará la estructura cognitiva neuronal para lograr el desarrollo de la inteligencia, expresada en la capacidad del individuo de ser autogestivo y de tener autocontrol; objetivos finales que persigue la teoría de la modificabilidad cognitiva estructural de Reuven Feuerstein (Feuerstein, Feuerstein, Falik y Rand, 2006).

Pilar Martín-Lobo (2016) habla de la visión que debe de existir acerca del estudiante bajo la perspectiva de la neuroeducación, concibiendo que la persona es "una unidad y la organización neurológica debe ser tenida en cuenta en diagnósticos y programas para que las actuaciones de los educadores y de los especialistas sean efectivas" (p. 25). Se debe de ajustar el modelo de planeación didáctica para tomar en cuenta las capacidades cognitivas del individuo y del grupo para realizar un ejercicio de aprendizaje significativo y exitoso.

Teóricamente se deben sentar las bases que apoyan este proyecto de investigación, teniendo un acercamiento a los conceptos que intervienen en la conformación de la fundamentación teórica, ya que dentro de las variaciones conceptuales existen también diferencias en cuanto al significado de dichos constructos, tal es el caso de la neurociencia cognitiva, la neurociencia del aprendizaje, la neuroeducación, 
la neuroanatomía, la neurofisiología, la neuropsicología, entre otras, que basan su trabajo en un área específica y visualizan de forma muy particular a la neurociencia, pero convergen en el trinomio cerebro-mente-conducta.

Sin embargo, el campo científico al que se refiere esta investigación se conoce como neuroeducación, que tiene como objetivo principal el mejoramiento de la práctica educativa a través del uso del conocimiento pedagógico y neurocientífico, que va a beneficiar tanto a estudiantes regulares como a alumnos con necesidades educativas especiales como las deficiencias cognitivas, auditivas, visuales o motoras. Algunas propuestas específicas que revisaremos más adelante para atender esta problemática la constituyen el Diseño Universal para el Aprendizaje (DUA) y la Teoría de la Modificabilidad Cognitiva Estructural (MCE), ya que en las últimas décadas:

Viene emergiendo una nueva ciencia, la Neuroeducación como una nueva línea de pensamiento y acción que tiene como principal objetivo acercar a los agentes educativos a los conocimientos relacionados con el cerebro y el aprendizaje, considerando la unión entre la Pedagogía, la Psicología Cognitiva y las Neurociencia (Campos, 2010, p. 10).

Una de las disciplinas que se gestaron como derivado de la neuroeducación es la neurodidáctica, que se ha establecido como el área que se ocupa del estudio del cerebro y de cómo es que funciona, con la finalidad de poder proponer mejoras en la dinámica pedagógica de los docentes actuales (D’Addario, 2019). Es entonces la neurodidáctica "la aplicación de conocimientos acerca de cómo funciona el cerebro y de cómo intervienen los procesos neurobiológicos en el aprendizaje, para ayudar a que éste sea más eficaz y óptimo" (Forés \& Ligioiz, 2009, p. 19). Aunado a lo anterior, Calzadilla (2017) establece que la neurodidáctica se constituye como el andamiaje fundamental de la neuropedagogía, la cual es la siguiente área de conocimiento surgida de esta fusión de ciencias, constituyéndose como la unión entre lo neurocientífico y lo pedagógico, proporcionando un "campo de aplicación teórico-práctico sobre el desarrollo mental, favorecido por nuevas técnicas no invasivas que permiten estudiar una función nerviosa en tiempo real, in vivo, de forma no traumática, indolora y precisa" (Ferreira, 2012, p. 36); tiene la posibilidad de ayudar a los docentes a comprender cómo es el proceso para los alumnos con diferentes problemas (D'Addario, 2019); por lo anterior es importante que la neuroeducación empiece a permear la práctica pedagógica para lograr consolidarse como "el campo interdisciplinario que se construye a partir de las conexiones entre neurociencia, cognición, psicología y educación, en un esfuerzo por crear una nueva ciencia del aprendizaje que transforme a la docencia” (Nouri \& Mehrmohammadi, 2013, p. 60).

Es importante entonces conocer la presencia de las materias relacionadas con la neuroeducación en los planes y programas de estudio para la formación y profesionalización docente a nivel de licenciatura y posgrado. En algunos otros países, como España (Zabalza \& Zabalza, 2018) y Cuba (Calzadilla, 2017), se han realizado diagnósticos similares al que presentamos en este documento, encontrando que, en el caso de Espańa, se requiere la incorporación de la neuroeducación en los planes y programas de estudio de futuros educadores y educadoras. Por su parte, Oscar Ovidio Calzadilla (2017) considera que en Cuba "la presencia del conocimiento de las Neurociencias en la formación de docentes tiene aún un nivel de transferencia limitado como consecuencia de la carente integración entre las Neurociencias y la Pedagogía" (p. 13); otro estudio realizado en Cuba demuestra que a partir de la información obtenida en la Universidad de Cienfuegos:

se comprobó que todavía es insuficiente el conocimiento que tienen, con respecto a los contenidos de la Neurociencia aplicables a la educación. Asimismo, se constató las deficiencias del tratamiento de dichos contenidos en los actuales Planes de Estudio y Programas de Disciplinas, de ahí que se identifica como problema científico la necesidad de incluir el tratamiento de la neurociencia en el contenido de la formación inicial de docentes. Entre los principales resultados se destaca la nula preparación recibida por los docentes en formación en cuanto a conoci- 
mientos neurocientíficos así como el insuficiente tratamiento del tema en las disciplinas y asignaturas que contribuyen a su formación profesional para enfrentar la actividad pedagógica (Jiménez, López y Herrera, 2019, p. 241).

Sin embargo la pertinencia de este estudio no solo se fundamenta en la cantidad de materias que existen dentro del currículo de los programas de licenciatura y posgrado para la formación de docentes, sino también en la percepción que tienen los docentes sobre el tema. En un estudio realizado en Ecuador se encontró que el $91.6 \%$ considera que los conocimientos en neuroeducación son relevantes para su quehacer profesional; el $45 \%$ consulta publicaciones periódicas referentes al tema; un $37 \%$ ha recibido formación en el área y el $97.8 \%$ muestra interés en temas de neuroeducación (Falquez \& Ocampo, 2018). Estas cifras son muy altas respecto a otros países, incluso si se comparan con México, ya que un estudio realizado en la Benemérita y Centenaria Escuela Normal del Estado de Sinaloa concluye que los docentes en formación "conocen la importancia y relevancia de que los docentes conozcan sobre la neurociencia y su implicación en la educación, debido a que ningún participante estuvo en desacuerdo en la posible inclusión de estos saberes en la formación normalista" (Aguilar, Conde y Hernández, 2019, p. 10) y proponen "incluir la neurociencia como parte de los cursos del trayecto optativo en la formación de los normalistas" (p. 12).

\section{Metodología}

\section{Objetivo}

Caracterizar los planes y programas de estudio de las instituciones formadoras de docentes en México que ofrecen formación en neurociencias.

\section{Preguntas de investigación}

¿En qué medida la neuroeducación forma parte de los planes y programas de formación y profesionalización docente en México?

¿Cuáles son los campos de estudio pertenecientes a la neuroeducación que predominan en los planes y programas de formación y profesionalización docente en México?

Para la realización de este estudio se utilizó una metodología cuantitativa con un enfoque no experimental de alcance descriptivo y con diseño transeccional. La muestra es censal, constituida por 2,891 planes y programas de estudio de licenciatura y posgrado relacionados con la formación, actualización o profesionalización docente en México.

Para la recopilación de la información se llevó a cabo una técnica de descarte, en la que se revisó la base de datos de la Asociación Nacional de Universidades e Instituciones de Educación Superior (ANUIES) usando el registro por Estado con el que la institución cuenta. En primer lugar, se revisó el sitio web de cada una de las instituciones de educación superior para identificar aquellos programas que estuvieran relacionados con la formación y profesionalización docente; una vez localizado el programa pertinente a este estudio, se revisó el plan de estudios o la malla curricular para registrar en un formato prediseñado los datos de aquellas materias relacionadas con la neuroeducación y los programas e instituciones a los que pertenece.

Para la recopilación de la información se utilizó una ficha de registro como instrumento, diseñado como una hoja electrónica en donde se capturaron los datos para el análisis descriptivo del objeto de estudio constituido por los indicadores: nombre de la institución, el tipo de financiamiento, el Estado, la ciudad, el nombre y nivel del programa y por último la o las materias relacionadas con neuroeducación que se encontraron dentro de la malla curricular.

La información recopilada se analizó en base a diferentes variables que aportaron información relevante a este estudio, revisando la presencia de las materias neuroeducativas por nivel educativo, por Estado y por tipo de financiamiento. Los resultados permitieron, en primer lugar, tener un diagnóstico de todos los programas de formación, actualización y profesionalización docente que existen en México y, posteriormente, un análisis de aquellos que contienen materias relacionadas con la neuroeducación en su currículo. 


\section{Resultados}

\section{Panorama general de los programas de forma- ción y profesionalización docente en México}

La investigación se realizó a partir de los registros del Directorio Nacional de la ANUIES. La base de datos de esta organización permitió revisar las instituciones de educación superior por desagregada, por cada uno de los Estados, utilizando los hipervínculos hacia los sitios web de dichas escuelas; sin embargo, algunos de estos sitios se encontraron dados de baja, inactivos o en mantenimiento, en tal caso se procedió como segunda fuente de información a consultar el Sistema de Información Básica de Educación Normal (SIBEN) para el caso de las Escuelas Normales, Centros de Actualización del Magisterio e instituciones educativas incorporadas a la Dirección General de Educación Superior para el Magisterio (DGESUM, antes DGESPE); y como tercer fuente de información se buscó el portal de Facebook oficial de la institución, con el fin de conocer los diferentes programas de estudio y consultar los planes y programas para encontrar las materias relacionadas con la neurociencia, la neuroeducación o la neurodidáctica.

De esta manera se revisaron 2,891 planes de estudio relacionados con la formación de docentes en todas sus áreas, provenientes de 650 instituciones diferentes, de los cuales el $54 \%(1,558)$ es de nivel licenciatura, el 5\% (143) pertenece al nivel de especialidad, el 33\% (949) es de nivel maestría y un 8\% (241) de nivel doctorado. De la totalidad de programas revisados, el $41 \%$ corresponde a instituciones de carácter público y el resto (59\%) a instituciones privadas. Un dato relevante se refiere al Estado de la República que tiene mayor número de programas de estudio en formación y actualización de docentes: Veracruz, con 254 programas, que representan el 8\% del total; el Estado con menos programas es Colima, con solamente 23 programas, que representan un 1\% del total. La ciudad que más programas de formación y profesionalización de docentes tiene es Ciudad de México, con 187 programas disponibles.

\section{Programas de licenciatura referentes a la forma- ción docente en México}

Respecto a la información estadística general del segmento correspondiente al nivel licenciatura, de los 1,558 programas encontrados disponibles en México, exactamente el 50\% (779) es de sostenimiento público y la otra mitad pertenece al régimen de instituciones privadas; de ellos, el Estado de Veracruz es el que más programas de formación inicial tiene, con un total de 118 programas, seguido por el Estado de México, con 117; Puebla, con 97; Ciudad de México, con 84; Baja California, con 73; hasta llegar al Estado con menor número, Tlaxcala, con tan solo 1 programa de licenciatura para la formación inicial. Cabe mencionar que, del total de programas, 36 de ellos están disponibles en la modalidad de educación virtual. A continuación, en la Tabla 1 quedará representado lo antes expuesto.

\section{Tabla 1}

Programas de licenciatura en formación de docentes ofertados en México

\begin{tabular}{ccc}
\hline & Programas de licenciatura ofertados & Porcentaje \\
\hline Escuelas normales públicas & 407 & 26 \\
\hline Escuelas normales privadas & 65 & 4 \\
\hline Centros de actualización del magisterio & 12 & 1 \\
\hline Unidades de la Universidad Pedagógica Nacional & 164 & 11 \\
\hline Universidades e instituciones privadas & 714 & 46 \\
\hline Universidades e instituciones públicas & 196 & 13 \\
\hline
\end{tabular}


En la formación de docentes en México a nivel licenciatura predomina la Licenciatura en Pedagogía, con 251 programas disponibles (16.1\%), seguida por la Licenciatura en Educación Primaria, con 171 programas disponibles (11\%); posteriormente, encontramos la Licenciatura en Educación Preescolar, con 146 programas (9.4\%); la Licenciatura en Ciencias de la Educación representa el 8.4\%, con 131 programas; y la Licenciatura en Comunicación está presente en 70 programas, que representan un $4.5 \%$. El resto de la muestra no es representativo.

Dentro de las categorías en las que se pueden organizar los programas de licenciatura relacionados con la formación docente destaca la categoría de Pedagogía, que reúne a 257 programas (16.5\%); luego, encontramos la categoría de Educación, con 201 programas (12.9\%); la que se refiere a Educación primaria, con 172 programas (11\%); la categoría de Educación preescolar reúne 146 programas $(9.4 \%)$ y la categoría de Educación física reúne a 112 programas (7.2\%).

\section{Programas de licenciatura con materias relacio- nadas con la neuroeducación}

De los 1,558 programas de licenciatura en México que se relacionan con la formación de docentes, el 19.6\% (306) contiene dentro de sus planes y programas de estudio una o más materias que se relacionan con la neuroeducación; de estos programas, el $60 \%$ pertenece a instituciones públicas. Cabe mencionar que, ante la imposibilidad de acceder a los temarios específicos de cada materia, el análisis que se presenta a continuación es solamente un diagnóstico cuantitativo del número y el tipo de materias neuroeducativas que están siendo parte del currículo de la formación de docentes en México.
Para empezar el análisis de la presencia de la neuroeducación en la formación docente, se inicia con la revisión de la oferta que tiene cada Estado, encontrando que la entidad que tiene mayor número de programas con materias de neuroeducación a nivel licenciatura es el Estado de México, con 41 programas; seguido por Chihuahua, con 22; Puebla, con 21; Ciudad de México, con 14; Nuevo León, Querétaro y Veracruz, con 12 cada uno; Baja California, con 11; Morelos, con 9; Baja California Sur, Durango, Guanajuato y Tamaulipas, con 9 programas cada uno; Chiapas, Jalisco, Oaxaca, San Luis Potosí, Sonora y Yucatán, con 8 programas cada uno; Coahuila, Tabasco y Zacatecas, con 7 programas; Campeche, Colima, Michoacán, Quintana Roo y Sinaloa, con 5 programas; en modalidad virtual, Aguascalientes, Guerrero e Hidalgo, con 4 programas; Tlaxcala, con 3 programas y Nayarit con 2.

Entre los programas con materias neuroeducativas que destacan encontramos la Licenciatura en Psicopedagogía, que representa un 15.7\%, Licenciatura en Educación Secundaria (14.4\%), Licenciatura en Educación Física (11.4\%), Licenciatura en Inclusión Educativa (11.1\%) y Licenciatura en Pedagogía con (9.5\%); a partir de ahí, 44 programas más comparten el resto de la distribución, pero cuentan con muy baja representatividad. Los programas de licenciatura que destacan por tener el mayor número de materias de neuroeducación (ver Tabla 2) pertenecen a la Universidad Guadalupe Victoria, en Campeche; el Colegio Esparza, en Puebla y la Universidad La Salle Noroeste, en Sonora; las tres instituciones tienen el programa de Licenciatura en Psicología Educativa, sin embargo, difieren en las materias ofertadas. 
Tabla 2

Programas de licenciatura con materias de neuroeducación

\begin{tabular}{|c|c|c|c|c|}
\hline Institución & Programa & Materia 1 & Materia 2 & Materia 3 \\
\hline $\begin{array}{c}\text { Universidad Guadalupe } \\
\text { Victoria }\end{array}$ & $\begin{array}{c}\text { Licenciatura en } \\
\text { Psicología Educativa }\end{array}$ & Neuropsicología & $\begin{array}{c}\text { Programación } \\
\text { Neurolingüística I }\end{array}$ & $\begin{array}{c}\text { Programación } \\
\text { Neurolingüística II }\end{array}$ \\
\hline Colegio Esparza & $\begin{array}{c}\text { Licenciatura en } \\
\text { Psicología Educativa }\end{array}$ & Neuropsicología & $\begin{array}{c}\text { Evaluación } \\
\text { Neuropsicológica I }\end{array}$ & Neuropsicológica II \\
\hline $\begin{array}{c}\text { Universidad La Salle } \\
\text { Noroeste }\end{array}$ & $\begin{array}{c}\text { Licenciatura en } \\
\text { Psicología Educativa }\end{array}$ & $\begin{array}{c}\text { Bases biológicas y desarrollo } \\
\text { neurobiológico }\end{array}$ & Neuropsicología Básica & Neuroeducación \\
\hline
\end{tabular}

Respecto a las materias encontradas en los programas de licenciatura, la representatividad mayor la tiene la materia Neurociencia en la Adolescencia, con un 26\%, y que corresponde a programas dentro de las instituciones pertenecientes a la DGESUM. Enseguida se posiciona la materia Desarrollo Neuropsicológico, con un $11 \%$ y, con el mismo porcentaje, la materia Trastornos Neuropsicológicos del Aprendizaje; la materia Neurociencia de la Infancia y la Juventud, con un 10\%; Neurociencia de la Conducta, con 6\%; Neurobiología del Desarrollo, con 5\%; Motivación y Emoción, 3\%; Neurofisiología, 3\%; Neuropsicología, 3\%; Neurociencias, 2\%; Programación Neurolingüística, 2\%; y con un $1 \%$ o menos en cada materia Neuroanatomía Funcional, Bases Neuropsicológicas del Aprendizaje en la Educación Inicial, Fundamentos Neuropsicológicos, Neuroeducación, Neurofisiología del Aprendizaje, Neuropsicofisiología e Intervención Educativa, Neuropsicología del Aprendizaje, Aprendizaje y Neurodesarrollo, Aprendizaje y sus Bases Neurológicas, Bases Neurológicas de la Conducta, Neurociencias y Educación, Neurofisiología y Neuroanatomía, Neurolingüística, Re-Educación de Trastornos Cognitivos, Aprendizaje y Cerebro, Neurodesarrollo Típico y Atípico, Base Neuropsicológica del Aprendizaje en la Educación Inicial, Bases Biológicas y Desarrollo Neurológico, Bases de Neuroanatomía, Bases de Neurofisiología, Bases Neuropsicológicas de la Conducta I, Bases Neuropsicológicas de la Conducta II, Evaluación Neuropsicológica I, Evaluación Neuropsicológica II, Fundamentos Neuropsicológicos de la Conducta, Neuroanatomía, Neurobiología del Aprendizaje y
Alteraciones Asociadas, Neurociencia Educativa I, Neurociencia Educativa II, Neurociencias Aplicadas a La Educación, Neurociencias y Aprendizaje, Neurocognición, Neurodesarrollo Aplicado a la Educación, Neurodesarrollo y Aprendizaje, Neurodidáctica, Neurofisiología del Comportamiento, Neurolingüística y Alteraciones del Lenguaje, Neuromotricidad, Neuropsicología Básica, Neurodesarrollo, Psicolingüística y Neurolingüística, Psiconeurología del Adulto y Psiconeurología Infantil.

Debido al gran número de materias y con la necesidad de reducir las categorías, se procedió a hacer un agrupamiento de estas para tener un panorama más sintético sobre el tipo de materias que se están impartiendo en los programas de formación inicial docente; es de esta manera que las materias que se refieren a las Neurociencias representan un $46.3 \%$, las que se agrupan en Neuropsicología son un $18.7 \%$, la categoría de Trastornos Neuropsicológicos representa un 11.4\%; Neurobiología, 6.0\%; Neurofisiología, 4.9\%; Motivación y Emoción, 3.0\%; Programación Neurolingüística, 1.9\%; Neuroanatomía, 1.6\%; con $1 \%$ Neurodesarrollo y el resto de la muestra no es representativo.

\section{Programas de especialidad relacionados con la formación docente en México}

El segmento que corresponde a los programas de especialidad (ver Tabla 3) representa el 5\% de la oferta total de formación y profesionalización docente en México, lo que significa que existen 143 programas disponibles, de los cuales el $40 \%$ es de sostenimiento 
público y el resto $(60 \%)$ corresponde a instituciones privadas. Dentro de los programas de especialidad, el Estado que más programas tiene es la Ciudad de México, con 25 programas, seguido por el Estado de México, con 19 y Veracruz, con 18, encontrando algunos casos con ningún programa de especialidad para la profesionalización de los maestros, como Baja California Sur, Colima, Guerrero, Michoacán, Nayarit, San Luis Potosí, Sinaloa, Tamaulipas y Tlaxcala. Es importante seńalar que 8 programas de especialidad se ofertan en la modalidad virtual.

\section{Tabla 3}

Programas de especialidad para profesionalización de docentes ofertados en México

\begin{tabular}{ccc}
\hline & $\begin{array}{c}\text { Programas de } \\
\text { especialidad } \\
\text { ofertados }\end{array}$ & Porcentaje \\
\hline $\begin{array}{c}\text { Escuelas normales } \\
\text { públicas }\end{array}$ & 7 & 5 \\
\hline $\begin{array}{c}\text { Escuelas normales } \\
\text { privadas }\end{array}$ & 0 & 0 \\
\hline $\begin{array}{c}\text { Centros de actualización } \\
\text { del magisterio }\end{array}$ & 5 & 15 \\
\hline $\begin{array}{c}\text { Unidades de la } \\
\text { Universidad Pedagógica } \\
\text { Nacional }\end{array}$ & 22 & 60 \\
\hline $\begin{array}{c}\text { Universidades e } \\
\text { instituciones privadas }\end{array}$ & 86 & 16 \\
\hline $\begin{array}{c}\text { Universidades e } \\
\text { instituciones públicas }\end{array}$ & 23 & \\
\hline
\end{tabular}

Dentro de estos programas no existe una preponderancia significativa de ninguno de ellos, ya que la Especialidad en Competencias para la Enseñanza de las Artes en Educación Básica y la Especialidad en Docencia son los dos planes que tienen mayor presencia, con un $9 \%$ cada uno. Agrupando las especialidades en categorías encontramos que el tipo de especialidad que predomina en México es Matemáticas, con 14\% de representatividad; Competencias, con 14\%; Inclusión, 9\%; Género, 9\%; Docencia, 9\%; y el resto tiene una representatividad no significativa.

\section{Programas de especialidad con materias rela- cionadas con la neuroeducación}

Los programas de especialidad en México que buscan la profesionalización de los docentes tienen un índice muy bajo de materias relacionadas con neuroeducación (ver Tabla 4), encontrando que tan solo uno de los 22 programas incluye dentro de su mapa curricular materias neuroeducativas: la Especialidad en Neuropsicología Educativa, impartida en la Universidad Panamericana campus Aguascalientes; incluye las materias de Bases Neuromorfofisiológicas del Comportamiento, Fundamentos de Neuropsicología, Neurofilosofía y Bioética, Fundamentos de Evaluación Neuropsicológica y Taller de Evaluación Neuropsicológica.

\section{Tabla 4}

Programas de especialidad con materias de neuroeducación

\begin{tabular}{|c|c|c|c|}
\hline Institución & Adscripción & Especialidad & Materia(s) \\
\hline $\begin{array}{l}\text { Universidad } \\
\text { Panamericana }\end{array}$ & Privada & $\begin{array}{c}\text { Especialidad } \\
\text { en Neurop- } \\
\text { sicología } \\
\text { Educativa }\end{array}$ & $\begin{array}{c}\text { Bases Neuromor- } \\
\text { fofisiológicas del } \\
\text { Comportamiento, } \\
\text { Fundamentos } \\
\text { de Neuropsico- } \\
\text { logía, Neurofilo- } \\
\text { sofía y Bioética, } \\
\text { Fundamentos } \\
\text { de Evaluación } \\
\text { Neuropsicológica, } \\
\text { Taller de Evalua- } \\
\text { ción Neuropsico- } \\
\text { lógica }\end{array}$ \\
\hline
\end{tabular}

\section{Programas de maestría relacionados con la formación docente en México}

Los programas de maestría mexicanos que profesionalizan a los docentes representan el 33\% (949) del total de los programas de formación y profesionalización (ver Tabla 5), de los cuales el 30\% (289) es de carácter público y el 70\% (660) pertenece a 
instituciones privadas. El Estado que presenta más programas de maestría relacionada con la profesionalización de maestros es Veracruz, con 87 programas; Ciudad de México, con 74 programas; Puebla, con 68; Chihuahua, con 57; Estado de México, con 52; y el Estado que menos programas reporta es Colima, con tan solo 6 programas registrados. Los programas ofertados en modalidad virtual son 35 .

\section{Tabla 5}

Programas de maestría para profesionalización docente ofertados en México

\begin{tabular}{ccc}
\hline & $\begin{array}{c}\text { Programas } \\
\text { de maestría } \\
\text { ofertados }\end{array}$ & Porcentaje \\
\hline Escuelas normales públicas & 37 & 4 \\
\hline Escuelas normales privadas & 8 & 1 \\
\hline $\begin{array}{c}\text { Centros de actualización del } \\
\text { magisterio }\end{array}$ & 6 & 1 \\
\hline $\begin{array}{c}\text { Unidades de la Universidad } \\
\text { Pedagógica Nacional }\end{array}$ & 141 & 15 \\
\hline $\begin{array}{c}\text { Universidades e instituciones } \\
\text { privadas }\end{array}$ & 652 & 69 \\
\hline $\begin{array}{c}\text { Universidades e instituciones } \\
\text { públicas }\end{array}$ & 105 & 11 \\
\hline
\end{tabular}

En los programas de maestría en México dirigidos a la profesionalización docente predomina la Maestría en Educación, ofertada 231 veces, representando el 24.3\% del total; le sigue la Maestría en Ciencias de la Educación, con un 5.5\%; la Maestría en Educación Básica, con un 5.1\%; la Maestría en Educación Media Superior, con el 3.5\%; la Maestría en Pedagogía, con el 2.5\%; y la Maestría en Docencia, también con un 2.5\%; el resto se lo dividen 59 programas de maestría diferentes con una representatividad muy baja.

Agrupando estas maestrías en categorías afines, podemos tener una idea de hacia dónde se encuentra la tendencia en cuanto a la formación de posgrado en este nivel. En principio, destaca la categoría de Ciencias de la Educación, que ocupa el 32.9\% de todos los programas, seguida por la de Educación Básica, con un 5.7\%; luego, la de Educación Media Superior, con el 4.3\%; el resto tiene una representatividad demasiado baja.

\section{Programas de maestría con materias relacio- nadas con la neuroeducación}

De los 949 programas de maestría registrados en México, tan solo el 4\% (39) cuenta con una o más materias relacionadas con la neuroeducación; el Estado que más programas tiene es Veracruz, con 6. Hay una gran cantidad de Estados donde no se cuenta con programas de maestría con materias relacionadas con la neuroeducación. A continuación, en la Tabla 6 se presenta la información completa de los programas de maestría que responden a las características de tener materias neuroeducativas.

\section{Tabla 6}

Programas de maestría con materias relacionadas con la neuroeducación

\begin{tabular}{ccccc}
\hline Institución & Estado & Ciudad & Maestría & Materia(s) \\
\hline Universidad IEU & Campeche & Campeche & $\begin{array}{c}\text { Maestría En Educación } \\
\text { Especial }\end{array}$ & $\begin{array}{c}\text { Bases Neurológicas del Desarrollo } \\
\text { Humano }\end{array}$ \\
\hline Universidad IEU & Campeche & Campeche & $\begin{array}{c}\text { Maestría En } \\
\text { Psicopedagogía }\end{array}$ & Neuropsicología \\
\hline $\begin{array}{c}\text { Universidad } \\
\text { Panamericana }\end{array}$ & CDMX & CDMX & $\begin{array}{c}\text { Maestría En } \\
\text { Neuropsicología y } \\
\text { Educación }\end{array}$ & $\begin{array}{c}\text { Niveles Táctiles y Neuromotores, } \\
\text { Procesos Neurolingüísticos } \\
\text { y Niveles de Aprendizaje, } \\
\text { Desarrollo de las Inteligencias } \\
\text { Múltiples, Creatividad }\end{array}$ \\
\hline
\end{tabular}




\begin{tabular}{ccccc}
\hline $\begin{array}{c}\text { Centro Mexicano } \\
\text { de Programación } \\
\text { Neurolingüística }\end{array}$ & CDMX & CDMX & $\begin{array}{c}\text { Maestría en Programación } \\
\text { Neurolingǘstica }\end{array}$ & $\begin{array}{c}\text { Programación Neurolingüística } \\
\text { en Educación, Programación } \\
\text { Neurolingüística en } \\
\text { Organizaciones, Programación } \\
\text { Neurolingüística y Salud }\end{array}$ \\
\hline $\begin{array}{c}\text { Universidad Justo } \\
\text { Sierra }\end{array}$ & CDMX & CDMX & Maestría en Psicopedagogía & $\begin{array}{c}\text { Bases Neurológicas del } \\
\text { Aprendizaje }\end{array}$ \\
\hline $\begin{array}{c}\text { Universidad Linda } \\
\text { Vista }\end{array}$ & Chiapas & Solistahuacán & $\begin{array}{c}\text { Maestría en Docencia y } \\
\text { Gestión }\end{array}$ & Trastornos del Neurodesarrollo \\
\hline $\begin{array}{c}\text { Universidad Pablo } \\
\text { Guajardo Chávez }\end{array}$ & Chiapas & Tuxtla Gutiérrez & Maestría en Psicopedagogía & Neuropsicología \\
\hline
\end{tabular}

Centro de

Especialidades en

Desarrollo y Educación
Maestría en Rehabilitación de Personas con

Chihuahua Chihuahua
Alteraciones del Lenguaje y la Audición
Neurolingüística

\begin{tabular}{ccccc}
\hline Universidad IEU & En Línea & En Línea & $\begin{array}{c}\text { Maestría en Educación } \\
\text { Especial }\end{array}$ & $\begin{array}{c}\text { Bases Neurológicas del Desarrollo } \\
\text { Humano }\end{array}$ \\
\hline Universidad IEU & En Línea & En Línea & Maestría en Psicopedagogía & Neuropsicología \\
\hline Universidad Ítaca & En Línea & En Línea & $\begin{array}{c}\text { Maestría en Ciencias de la } \\
\text { Educación }\end{array}$ & $\begin{array}{c}\text { Avances de las Neurociencias } \\
\text { Aplicadas a la Educación }\end{array}$ \\
\hline $\begin{array}{c}\text { Instituto Pedagógico de } \\
\text { Estudios de Posgrado }\end{array}$ & Guanajuato & Celaya & $\begin{array}{c}\text { Maestría en Educación } \\
\text { Inicial y Preescolar }\end{array}$ & Programación Neurolingüística \\
\hline
\end{tabular}

Universidad Mexicana
de Educación a
Guanajuato
León
Maestría en Psicopedagogía
Neuropsicología y Educación

Distancia

$\begin{array}{ccc}\text { Universidad } & \text { Maestría en } \\ \text { Panamericana } & \text { Galisco } & \text { Neuropsicología y } \\ \text { Educación }\end{array}$

Niveles Táctiles y Neuromotores,

Procesos Neurolingüísticos

y Niveles de Aprendizaje,

Desarrollo de las Inteligencias

Múltiples, Creatividad

\begin{tabular}{|c|c|c|c|c|}
\hline $\begin{array}{c}\text { Universidad } \\
\text { Panamericana }\end{array}$ & Jalisco & Guadalajara & Maestría en Pedagogía & Neurociencia y Aprendizaje \\
\hline $\begin{array}{l}\text { Universidad Marista } \\
\text { Guadalajara }\end{array}$ & Jalisco & Guadalajara & $\begin{array}{c}\text { Maestría en Estrategias de } \\
\text { Aprendizaje }\end{array}$ & Neuroeducación \\
\hline $\begin{array}{c}\text { Escuela Particular } \\
\text { Normal Superior del } \\
\text { Estado }\end{array}$ & Morelos & Cuernavaca & $\begin{array}{l}\text { Maestría en Docencia y } \\
\text { Ambientes Virtuales }\end{array}$ & Programación Neurolingüística \\
\hline $\begin{array}{c}\text { Escuela Particular } \\
\text { Normal Superior del } \\
\text { Estado }\end{array}$ & Morelos & Cuernavaca & $\begin{array}{c}\text { Maestría en Competencias } \\
\text { Docentes }\end{array}$ & Programación Neurolingüística \\
\hline $\begin{array}{c}\text { Escuela Normal } \\
\text { de Especialización } \\
\text { Humberto Ramos } \\
\text { Lozano }\end{array}$ & Nuevo León & Monterrey & $\begin{array}{c}\text { Maestría en Inclusión } \\
\text { Educawtiva }\end{array}$ & $\begin{array}{c}\text { Neurociencia y Estilos de } \\
\text { Aprendizaje }\end{array}$ \\
\hline
\end{tabular}




\begin{tabular}{|c|c|c|c|c|}
\hline $\begin{array}{l}\text { Universidad de } \\
\text { Monterrey }\end{array}$ & Nuevo León & $\begin{array}{l}\text { San Pedro Garza } \\
\text { García }\end{array}$ & $\begin{array}{l}\text { Maestría en Ciencias de la } \\
\text { Educación }\end{array}$ & $\begin{array}{c}\text { Didáctica para el Aprendizaje } \\
\text { Basada en la Neurociencia y la } \\
\text { Psicología Moderna }\end{array}$ \\
\hline Universidad IEU & Oaxaca & Oaxaca & $\begin{array}{l}\text { Maestría en Educación } \\
\text { Especial }\end{array}$ & $\begin{array}{c}\text { Bases Neurológicas del Desarrollo } \\
\text { Humano }\end{array}$ \\
\hline Universidad IEU & Oaxaca & Oaxaca & Maestría en Psicopedagogía & Neuropsicología \\
\hline $\begin{array}{l}\text { Universidad Regional } \\
\text { del Sureste }\end{array}$ & Oaxaca & Oaxaca & $\begin{array}{c}\text { Maestría en Psicología } \\
\text { Educativa }\end{array}$ & $\begin{array}{c}\text { Neuropsicología y } \\
\text { Comportamiento Humano }\end{array}$ \\
\hline Universidad IEU & Puebla & Puebla & $\begin{array}{l}\text { Maestría en Educación } \\
\text { Especial }\end{array}$ & $\begin{array}{c}\text { Bases Neurológicas del Desarrollo } \\
\text { Humano }\end{array}$ \\
\hline Universidad IEU & Puebla & Puebla & Maestría en Psicopedagogía & Neuropsicología \\
\hline $\begin{array}{l}\text { Instituto de Estudios } \\
\text { Superiores del Estado } \\
\text { de Puebla }\end{array}$ & Puebla & $\begin{array}{l}\text { Santiago } \\
\text { Miahuatlán }\end{array}$ & $\begin{array}{c}\text { Maestría en Ciencias del } \\
\text { Aprendizaje }\end{array}$ & $\begin{array}{c}\text { Aprendizaje Basado } \\
\text { enNeurociencia, Programación } \\
\text { Neurolingüística }\end{array}$ \\
\hline $\begin{array}{l}\text { Universidad La Salle } \\
\text { Noroeste }\end{array}$ & Sonora & Ciudad Obregón & Maestría en Educación & $\begin{array}{c}\text { Bases Neurofisiológicas del } \\
\text { Aprendizaje }\end{array}$ \\
\hline Universidad IEU & Tabasco & Villahermosa & $\begin{array}{l}\text { Maestría en Educación } \\
\text { Especial }\end{array}$ & $\begin{array}{c}\text { Bases Neurológicas del Desarrollo } \\
\text { Humano }\end{array}$ \\
\hline Universidad IEU & Tabasco & Villahermosa & Maestría en Psicopedagogía & Neuropsicología \\
\hline $\begin{array}{l}\text { Universidad Alfa y } \\
\text { Omega }\end{array}$ & Tabasco & Villahermosa & $\begin{array}{l}\text { Maestría en Neurociencia } \\
\text { Aplicada a la Psicología } \\
\text { Educativa y Organizacional }\end{array}$ & $\begin{array}{l}\text { Neurociencia y Aprendizaje, } \\
\text { Neurociencia y Psicopatologías }\end{array}$ \\
\hline $\begin{array}{l}\text { Universidad del } \\
\text { Noroeste, A.C. }\end{array}$ & Tamaulipas & Tampico & $\begin{array}{c}\text { Maestría en Educación } \\
\text { Especial }\end{array}$ & Neurodesarrollo Infantil \\
\hline $\begin{array}{l}\text { Universidad del } \\
\text { Noroeste, A.C. }\end{array}$ & Tamaulipas & Tampico & Maestría en Psicopedagogía & $\begin{array}{c}\text { Neurodesarrollo, Neuropsicología } \\
\text { de los Procesos de Aprendizaje, } \\
\text { Neuropsicología de la Lectura, } \\
\text { Escritura y el Cálculo. }\end{array}$ \\
\hline $\begin{array}{l}\text { Universidad Autónoma } \\
\text { de Tlaxcala }\end{array}$ & Tlaxcala & Tlaxcala & $\begin{array}{l}\text { Maestría en Educación } \\
\text { Especial }\end{array}$ & $\begin{array}{c}\text { Evaluación Neuropsicológica en } \\
\text { el Contexto Escolar, Modelos } \\
\text { y Estrategias de Intervención } \\
\text { Neuropsicológica para el } \\
\text { Aprendizaje Escolar }\end{array}$ \\
\hline Universidad IEU & Veracruz & Veracruz & $\begin{array}{l}\text { Maestría en Educación } \\
\text { Especial }\end{array}$ & $\begin{array}{c}\text { Bases Neurológicas del Desarrollo } \\
\text { Humano }\end{array}$ \\
\hline Universidad IEU & Veracruz & Veracruz & Maestría en Psicopedagogía & Neuropsicología \\
\hline
\end{tabular}




\begin{tabular}{|c|c|c|c|c|}
\hline $\begin{array}{l}\text { Centro de Estudios } \\
\text { Universitarios }\end{array}$ & Veracruz & Veracruz & Maestría en Educación & Neuroeducación \\
\hline $\begin{array}{l}\text { Centro de Estudios } \\
\text { Superiores de Veracruz }\end{array}$ & Veracruz & Veracruz & Maestría en Educación & Neuroeducación \\
\hline $\begin{array}{c}\text { Universidad Pedagógica } \\
\text { Veracruzana }\end{array}$ & Veracruz & Xalapa & $\begin{array}{l}\text { Maestría en Docencia para } \\
\text { la Educación Superior }\end{array}$ & $\begin{array}{c}\text { Tendencias de Aprendizaje; } \\
\text { Neuropedagogía }\end{array}$ \\
\hline $\begin{array}{l}\text { Universidad Popular } \\
\text { Autónoma de Veracruz }\end{array}$ & Veracruz & Xalapa & $\begin{array}{c}\text { Maestría en } \\
\text { Neuroeducación }\end{array}$ & $\begin{array}{c}\text { Neuroeducación, } \\
\text { Neuropsicología Cognitiva, } \\
\text { Neurodidáctica, Neuropsicología, } \\
\text { Programación Neurolingüística }\end{array}$ \\
\hline
\end{tabular}

La materia más recurrente en los planes anteriores es la de Neuropsicología, que se repite en 8 programas, seguida por Bases Neurológicas del Desarrollo Humano, con 6 repeticiones; Programación Neurolingüística, con 5; Neuroeducación, con 4; Procesos Neurolingüísticos y Niveles de Aprendizaje, con 2; Niveles Tácticos y Neuromotores, con 2; Neurociencia y Aprendizaje, con 2; Desarrollo de las Inteligencias Múltiples, con 2; y con una aparición se encuentran las siguientes materias: Trastornos del Neurodesarrollo, Tendencias de Aprendizaje, Neuropedagogía, Programación Neurolingüística y Salud, Programación Neurolingüística en Organizaciones, Programación Neurolingüística en Educación, Neuropsicología y Educación, Neuropsicología y Comportamiento Humano, Neuropsicología de los Procesos de Aprendizaje, Neuropsicología de la Lectura, Escritura y el Cálculo, Neuropsicología Cognitiva, Neurolingüística, Neurodidáctica, Neurodesarrollo Infantil, Neurodesarrollo, Neurociencia y Psicopatologías, Neurociencia y Estilos de Aprendizaje, Modelos y Estrategias de Intervención Neuropsicológicas para el Aprendizaje Escolar, Evaluación Neuropsicológica en el Contexto Escolar, Didáctica para el Aprendizaje Basada en la Neurociencia y la Psicología Moderna, Bases Neurológicas del Aprendizaje, Bases Neurofisiológicas del Aprendizaje, Avances de las Neurociencias Aplicadas a la Educación y Aprendizaje Basado en Neurociencia.

La agrupación de las materias permite tener un panorama de las tendencias en neuroeducación que siguen los programas de maestría, encontrando que la categoría de Neuropsicología domina casi un tercio de los programas, con un 30.2\%; seguido de Neurociencia, con un 24.5\%; Programación Neurolingüística, con $15.1 \%$; el resto no tiene representatividad importante.

\section{Programas de doctorado relacionados con la formación docente en México}

El 8\% (241) de los programas de formación y profesionalización docente en México corresponde a estudios de doctorado (ver Tabla 7); de ellos, el 27\% pertenece a instituciones públicas; el resto, el 73\%, corresponde a programas de educación privada. El Estado que tiene mayor número de programas de doctorado es Ciudad de México, con 25 programas; seguido por Chiapas, con 23; Veracruz, con 21; Estado de México, con 17; Colima es el único Estado donde no se tiene registro de programas de doctorado. Cabe mencionar que, de la totalidad de doctorados, 10 se ofrecen de forma virtual. 
Tabla 7

Programas de doctorado para profesionalización docente ofertados en México

\begin{tabular}{ccc}
\hline & $\begin{array}{c}\text { Programas } \\
\text { de doctorado } \\
\text { ofertados }\end{array}$ & Porcentaje \\
\hline $\begin{array}{c}\text { Escuelas normales } \\
\text { públicas }\end{array}$ & 3 & 1.2 \\
\hline $\begin{array}{c}\text { Escuelas normales } \\
\text { privadas }\end{array}$ & 2 & 0.8 \\
\hline $\begin{array}{c}\text { Centros de } \\
\text { actualización del } \\
\text { magisterio }\end{array}$ & 0 & 0.0 \\
\hline $\begin{array}{c}\text { Unidades de } \\
\text { la Universidad } \\
\text { Pedagógica Nacional }\end{array}$ & 22 & 9.1 \\
\hline $\begin{array}{c}\text { Universidades e } \\
\text { instituciones privadas }\end{array}$ & 175 & 72.6 \\
\hline $\begin{array}{c}\text { Universidades e } \\
\text { instituciones públicas }\end{array}$ & 39 & 16.2 \\
\hline
\end{tabular}

En la formación de doctorado en México en el área de docencia predomina el Doctorado en Educación, con 137 programas (56.8\%); Doctorado en Ciencias de la Educación representa un 7.9\% (19); Doctorado en Desarrollo Educativo representa el 2.5\% (6); Doctorado en Innovación Educativa, un 2.1\% (5); el Doctorado en Investigación Educativa, 2.1 (55); Doctorado en Investigación e Intervención Educativa, con un 1.7\% (4); Doctorado en Desarrollo Educativo con Énfasis en Formación de Profesores representa un $1.2 \%$ (3), al igual que el Doctorado en Evaluación Educativa, con un 1.2\% (3). El resto de los programas no tiene representatividad importante. Dentro de las categorías en las que se pueden organizar los programas de doctorado destacan los que se agrupan en la categoría de Educación, los cuales suman 141 y representan el 60.52\%, los que pertenecen a Ciencias de la Educación representan el 8.58\% (20) y el resto de las categorías presenta porcentajes muy bajos.

\section{Programas de doctorado con materias relacio- nadas con la neuroeducación}

De los 241 programas de doctorado relacionados con la formación y profesionalización docente en México no se encontró ninguno con materias relacionadas con la neuroeducación.

\section{Conclusiones}

La información que produce el análisis de los planes y programas de estudio permite tener un panorama sobre en qué medida la neurociencia como disciplina científica y los diferentes campos de estudio que la conforman han logrado permear la formación y profesionalización docente. En cuanto al número de programas que incluyen a la neuroeducación dentro de su malla curricular se observa que, como un proceso natural, la mayor presencia de estas materias se encuentra en el nivel licenciatura, con 306 programas; seguido por los de maestría, con 39 programas y uno de especialidad, finalizando con una presencia nula de la neuroeducación en los programas de doctorado. Posiblemente la reciente aparición de la disciplina dentro del campo académico requiere de mayor tiempo para consolidarse y formar parte del máximo grado de estudios dentro de cualquier área formativa, el doctorado, lo cual corrobora la afirmación de Oscar Ovidio Calzadilla, quien establece que "en la actualidad la formación de docentes en el conocimiento neurocientífico se desarrolla de forma fragmentada y descontextualizada en el diseño curricular de planes y programas" (Calzadilla, 2017, p. 15).

En cuanto al tipo de financiamiento de las instituciones que incluyen a la neurociencia en los planes y programas de formación y profesionalización docente prevalecen las instituciones privadas, con un $100 \%$ en el nivel de especialidad y un $90 \%$ en el nivel de maestría, contra un $60 \%$ de sostenimiento público en el nivel licenciatura, lo cual provee de un indicador que revela que las instituciones privadas tienen un mayor interés en incorporar las nuevas tendencias pedagógicas como parte de su currículo para la profesionalización docente a nivel maestría y especialidad; 
es pues necesario que las formadoras de docentes públicas vean a "la neurociencia educativa como una disciplina común para que tanto neurociencias como educación se unan a fin de integrar procedimientos con métodos de comportamientos relacionados con el aprendizaje, a partir de lo cual se pueda configurar un modelo sinérgico multidisciplinar" (Barrios Tao, 2016, p. 401).

Es por esto que se concluye que los campos de estudio que conforman la disciplina de la neuroeducación tienen un amplio abanico de posibilidades, ya que existen materias relacionadas con la neuroeducación, pero que se enfocan en diferentes áreas, como la psicología, la educación física, la educación especial, la neurociencia cognitiva, etcétera, algunas veces de forma trasversal, en donde la neurociencia se combina con dos o más campos de estudio, como es el caso de la neuropsicopedagogía o la neuromorfofisiológía. Por lo anterior, es interesante observar que, en el caso de los programas de licenciatura, el campo que destaca es el de neurociencias, presente en un $46.3 \%$ de los planes y programas de estudio, lo cual indica que en la formación inicial la tendencia es capacitar a los docentes en aspectos no pedagógicos, agravando la brecha que existe entre las dos, lo científico y lo pedagógico, en una tendencia que Navarro identifica como el gran problema de nuestro sistema educativo, que:

se encarga de generar límites que dificultan la conexión entre las diferentes áreas; la metodología empleada, en muchos casos, sigue los mismos patrones que la recibida cuando éramos alumnos, una educación basada en la trasmisión de contenidos y alejada de la experimentación y la motivación (Navarro, 2018, p. 50).

En el caso del único programa de especialidad que cuenta con materias de neuroeducación, este se circunscribe dentro del campo de la neuropsicología; lo anterior invita a analizar la presencia de la neurodidáctica como el campo de estudio que más se relaciona con la práctica docente y que se encuentra más ausente en los planes de estudio en todos los niveles, como parte de la brecha que los investigadores han identificado entre la neurociencia y la aplicación práctica de los conocimientos en el aula. La neurodidáctica tiene una presencia de tan solo un 3.8\% de los programas de maestría con materias neuroeducativas, un $0.8 \%$ en las materias de licenciatura y nula presencia en los programas de especialidad y doctorado. Esto confirma lo establecido en múltiples investigaciones y artículos científicos relacionados con la neuroeducación (Ferreira, 2012), en donde se expresa una falta de investigación empírica que provea de estrategias neurodidácticas útiles para la labor docente y no solamente literatura sobre el tema (Ferreira y Gómez, 2019).

Cabe destacar que gran parte del aporte de programas con materias de neuroeducación lo realizan las escuelas normales, ya que los planes de estudio son comunes a nivel nacional, siendo incluso adoptados por algunas normales particulares, por lo que, en algunos casos, el programa de Licenciatura en Educación Secundaria, que tiene diferentes especializaciones, como telesecundaria, biología, español, física, formación cívica y ética, geografía, historia, inglés, matemáticas y química, mantiene en su tronco común una materia de neuroeducación; Neurociencia en la Adolescencia, que reporta un total de 97 programas y que representa un $26 \%$ de la presencia de la neuroeducación en todos los planes de licenciatura. Un caso similar se da con la Licenciatura en Educación Física, en donde encontramos la materia de Neurociencia de la Infancia y la Juventud, con presencia en 36 programas. La Licenciatura en Inclusión Educativa contiene las materias Desarrollo Neuropsicológico y Trastornos Neuropsicológicos del Aprendizaje, que impactan a 42 programas. La Licenciatura en Educación Especial contiene la materia Neurobiología del Desarrollo con presencia en 20 programas y, por último, la Licenciatura en Educación Inicial, que contiene la materia Bases Neuropsicológicas del Aprendizaje en la Educación Inicial, que impacta a 3 programas. Lo anterior revela que los Planes y Programas de Estudio que están bajo el subsistema de la DGESUM impactan a 138 programas, que significan un $57 \%$ de los programas de licenciatura con presencia de la neuroeducación en sus currículos.

El objetivo de esta investigación fue realizar un diagnóstico de la presencia de la neuroeducación en 
los programas de formación y profesionalización de docentes en México, para lo cual se hizo necesario realizar, en primer lugar, un diagnóstico general de los programas ofrecidos tanto para la formación inicial como para la profesionalización de los maestros mexicanos; sin embargo, para cerrar este primer estudio las conclusiones se refieren exclusivamente a los programas que tienen en su currículo materias relacionadas con la neuroeducación.

Una vez analizados los datos obtenidos de esta búsqueda documental, se concluye que la neuroeducación tiene poca presencia en los planes y programas de estudio de licenciatura, especialidad, maestría y doctorado relacionados con la formación y profesionalización docente, ya que, de 2,891 programas revisados, solamente 346 (12\%) cuentan con materias neuroeducativas, de los cuales 306 son de nivel licenciatura, 1 de especialidad, 39 de maestría y ninguno de doctorado.

La predominancia de la categoría de Neurociencias, con un 46\%, en los programas de licenciatura devela un interés en formar a los docentes en aspectos del funcionamiento del cerebro a nivel biológico y neuronal, respaldando los resultados y las afirmaciones de investigadores en neuroeducación, que señalan una brecha entre el conocimiento científico del funcionamiento del cerebro y su aplicación en el aula, ya que al tratarse de programas de formación y profesionalización docente sería esperable que la categoría predominante fuera neuroeducación o neurodidáctica, que le dará al maestro la oportunidad de "conocer las bases y principios neurobiológicos que implican el funcionamiento cerebral, su desarrollo cognitivo, ontogenético y afectivo, le permitirá a los docentes tener en sus manos un gran recurso para diseñar su práctica, soportada sobre bases científicas" (Soto, 2016, p. 67).

En el caso de los programas de maestría, las materias se agrupan y dan como resultado la categoría predominante de Neuropsicología, la cual tiene un aporte muy significativo para la neuroeducación al ser una de las tres partes que le dan origen (neurociencia - psicología - pedagogía); no obstante, encontramos que las categorías que se pueden referir a la aplicación práctica de las neurociencias en el aula, como lo son Neuroeducación y Neurodidáctica, alcanzan apenas un $11 \%$ de importancia.

Una vez realizado este diagnóstico, se abre la posibilidad de continuar con una investigación a profundidad de los programas de estudio que cuentan con materias neuroeducativas con el fin de analizar los temas, contenidos, autores y enfoques que se encuentran dentro de los diferentes cursos y las distintas materias, así como conocer una tendencia de la formación en neuroeducación en México, lo cual sería de gran utilidad para investigadores interesados en esta temática.

Respecto a la formación y profesionalización docente en México, se recomienda la búsqueda de estrategias en las que el enfoque neuroeducativo pueda integrarse a la formación inicial de los maestros a mediano plazo, teniendo la posibilidad de diseñar materias optativas, como es el caso de las escuelas normales, o crear programas de especialidad en neuroeducación, ya que ambas acciones no requieren de la modificación del plan de estudios y pueden ser implementadas con mayor inmediatez.

\section{Referencias}

Aguilar, M., Conde, C. y Hernández, M. (2019). Importancia de la inclusión de la neuropedagogía en la formación docente en las escuelas normales: diagnóstico BYCENES. Congreso Nacional de Investigación Sobre Educación Normal, 1-14.

Barrios Tao, H. (2016). Neurociencias, educación y entorno sociocultural. Educación y Educadores, 19(3), 395-415. https://doi.org/10.5294/edu. 2016.19.3.5

Calzadilla, O. O. (2017). La integración de las neurociencias en la formación inicial de docentes para las carreras de la educación inicial y básica: caso Cuba. Actualidades Investigativas en Educación, 17(2). https://doi.org/10.15517/ aie.v17i2.28709

Campos, A. L. (2010). Neuroeducación: uniendo las neurociencias y la educación en la búsqueda del desarrollo humano. La Educación, 143, 1-14. 
D’Addario, M. (2019). Educación y Neurociencia ( $1^{\text {a }}$ ed.). Safe Creative.

Falquez, J. F. y Ocampo, J. C. (2018). Del conocimiento científico al malentendido. Prevalencia de neuromitos en estudiantes ecuatorianos. Revista Iberoamericana de Educación, 78(1), 87-106.

Ferreira, R. y Gómez, L. (2019). ¿Por qué la neurociencia debería ser parte de la formación inicial docente? Sinergies Chili, 15, 45-56.

Ferreira, T. J. (2012). Neurociencia + Pedagogía = Neuropedagogía: Repercusiones e Implicaciones de los Avances de la Neurociencia para la Práctica Educativa. Universidad Internacional de Andalucía.

Feuerstein, R., Feuerstein, R., Falik, L. y Rand, Y. (2006). The Feuerstein Instrumental Enrichment Program. ICELP Publications.

Forés, A. y Ligioiz, M. (2009). Descubrir la Neurodidáctica: aprender desde, en y para la vida. Carrera Edició, S. L.

Jiménez, E. H., López, M. M. y Herrera, D. (2019). La Neurociencia en la formación inicial de docentes. Revista pedagógica de la Universidad de Cienfuegos, 15(67), 241-249.

Martín-Lobo, P. (2016). Procesos y Programas de Neuropsicología Educativa (1 ${ }^{\mathrm{a}} \mathrm{ed}$.). Ministerio de Educación de España: Centro Nacional de Investigación e Innovación (CNIIE).

MECD. (2016). Procesos y programas de neuropsicología educativa. Ministerio de Educación, Cultura y Deporte, España.

Navarro, V. (2018). Metodologías interdisciplinares como herramienta para motivar a alumnado de altas capacidades. Revista Iberoamericana de Educación, 78(1), 43-66.
Nouri, A. \& Mehrmohammadi, M. (2013). Defining the boundaries of neuroeducation as a field of study. Educational Research Journal, 1-25.

Pherez, G., Vargas, S. y Jeréz, J. (2018). Neuroaprendizaje, una propuesta educativa: herramientas para mejorar la praxis del docente. Civilizar Ciencias Sociales y Humanas, 18(34), 149-166. https://doi.org/10.22518/usergioa/jour/ $\operatorname{ccsh} / 2018.1 / \mathrm{a} 10$

Sánchez, S., Castro, L., Casas, J. A. y Vallejos, V. (2016). Análisis Factorial de las Percepciones Docentes sobre Diseño Universal de Aprendizaje. Revista Latinoamericana de Educación Inclusiva, 10(2), 135-149.

Soto, C. A. (2016). Relación entre las prácticas pedagógicas y las neurociencias: aportes al currículo de educación inicial. Universidad Pedagógica Nacional.

Varela, Vi., Montoya, D. M., Tamayo, L., Moscoso, O., Castellanos, C. G., Castro, P. A., González, L. y Zuluaga, J. B. (2011). Protocolo Neuropsicopedagógico en la Evaluación Multidimensional del Trastorno por Déficit Atencional/ Hiperactividad -Tdah-: Implementación De Una Experiencia Investigativa. Revista Latinoamericana de Estudios Educativos (Colombia), 7(2), 139-156.

Zabalza, M. A. y Zabalza, M. A. (2018). Neurociencias y Formación De Profesores Para La Educación Infantil. Revista Latinoamericana de Educación Infantil, 7(1), 78-85. 